\title{
3 Research Square \\ One-Pot Fabrication Cellulose/Silica \\ Composite Microcapsules and Their Application in Cotton Fabrics
}

\section{Ling Lin ( $\sim$ janegirl007@163.com )}

Yancheng Institute of Technology https://orcid.org/0000-0002-4370-4230

Chunxia Wang

Yancheng Institute of Technology

Haiyan Mao

Yancheng Institute of Technology

Huanling Wu

Yancheng Institute of Technology

Ziyin Li

Yancheng Institute of Technology

\section{Research Article}

Keywords: Cellulose, Microcapsules, Fabric coatings, Fragrance, Superhydrophobicity, UV protection

Posted Date: December 13th, 2021

DOI: https://doi.org/10.21203/rs.3.rs-1142143/v1

License: (c) (i) This work is licensed under a Creative Commons Attribution 4.0 International License. Read Full License

Version of Record: A version of this preprint was published at Cellulose on March 31st, 2022. See the published version at https://doi.org/10.1007/s10570-022-04508-7. 


\section{Abstract}

Research on multi-functional fabrics is an inevitable trend in the future development of textile field. Nevertheless, the key to the development of multifunctional fabric is how to solve the problem of contradictory function combination and how to achieve the multifunctions. In this work, a novel multifunctional fabric based on cellulose/silica microcapsules was developed by using green, facile and economical methods. Owing to the loaded essence and hydrophobic coupling reagent and UV absorber modified silica of microcapsules, the coated fabric not only exhibited slow-release property of fragrance, but also had excellent superhydrophobicity and ultraviolet (UV) protection. Furthermore, multifunctional fabric also displayed the durable superhydrophobicity and UV protection even after chemically or mechanically damaged. The development of mulfunctional fabrics largely depends on the development of textile functional finishing agent and finishing technology. Therefore, it is very important to develop new functional micrcapsules or finishing agent.

\section{Introduction}

With the rapid development of the textile industry and the continuous improvement of people's living standard, people's requirements for are not only just covering up against the cold, but also in many aspects (Bilisik and Demiryurek 2012; Bilisik and Yildirim 2014). Differentiated and functional textiles become the important direction of industrial development, such as antibacterial, dirt resistant, flame retardant, fragrance and ant-istatic electricity (Cheng et al. 2018; Hou et al. 2021; Jhatial et al. 2019; Li et al. 2021; Lv et al. 2020; Wang et al. 2019; Zhou et al. 2020), which are paid more attention by people today. Therefore, the textile finishing agent is the key to this kind of functional textiles development (Han and Gong 2021; Hu et al. 2020; Liu et al. 2021; Wang et al. 2020).

- Microcapsule technology is developing rapidly, and the organic combination of unique functions and textiles can bring more comfort to our life from the olfactory (Hong and Park 1999; Li et al. 2016; Moreira et al. 2020; Pak et al. 2019; Sun and Zhang 2001; Taguchi et al. 2007; Zhao et al. 2020), visual and psychological aspects (Cao and Yang 2014; Fei and Wu 2017; Soares-Latour et al. 2013; Son et al. 2014; Turan et al. 2021; Zhao et al. 2016). Scientific studies show that a lot of aromatics have the sedation, sterilization, hypnosis and health care properties. However, simply spraying the aromatic agent on the textile can not play a good role in the retention of fragrance, and its retention period is very short. The aromatics-loaded microcapsules have sustained release property, and the release rate of fragrance can be effectively controlled, so the finished textile with microcapsules can release fragrance for a long time (Hu et al. 2013; Liu et al. 2019). Microcapsules were finished on the textiles by spinning, padding or coating methods (Aracil et al. 2015). Elesini et al. prepared microcapsules with essential oil as core material and melamine formaldehyde as wall material (Elesini et al. 2017). And this microcapsule was successfully applied to the bow tie that can release the fragrance through friction. Chong et al. synthesized microcapsules containing 8hydroxyquinoline as corrosion inhibitor and clove oil as natural antibacterial agent by in situ polymerization (Chong et al. 2019). The prepared microcapsules have antibacterial ability against 
Escherichia coli, Vibrio coralli and Bacillus aesculatum. Iqbal et al. reported phase change materialloaded microcapsules, which were combined with intelligent monofilament fiber to successfully melt microcapsules into polypropylene monofilament by using the melting spinning process (Iqbal and Sun 2014). The latent heat of prepared composite monofilament fiber was $9.2 \mathrm{~J} / \mathrm{g}$. Microcapsule technology can be applied in many fields of textiles, mainly depending on the core material and wall material for different types of finishing, and thus the microcapsule technology needs to be diverse and efficient. However, aroma finishing focuses on the permanence and controllability of core material aroma release. Flame-retardant finishing tends to the flame-retardant efficiency of the core material, but has low requirements for the wall material. The emphasis of antibacterial finishing is the scope, duration and rate of bacteriostasis. The focus of multifunctional textile finishing is the sensitivity of wall materials of microcapsules, among which multifunctional or intelligent textile finishing should be the main development area of microcapsule technology in the future.

- Natural polymer materials come from nature and have biodegradability, biocompatibility and nontoxic side effects (Klemm et al. 2005; Medronho and Lindman 2015; Xie et al. 2009). Natural polymer materials (chitosan, sodium alginate, gelatin, etc.) can also be used to prepare microcapsules. The commonly used preparation methods of natural polymer microcapsules include template method, interfacial polymerization method, layer by layer assembly method, microchannel technology and so on (Lam et al. 2012; Yuen et al. 2012). Song et al. used template method to remove calcium carbonate and polypropylamine hydrochloride from the core layer and chitosan and sodium dextran sulfate from the shell layer by ethylenediamine tetraacetic acid, and then embedded $\mathrm{pH}$-sensitive dye (Song et al. 2014). The prepared microcapsule can detect pH ranging from 3.3 to 6.5. Cui et al. prepared microcapsules of chitosan and hyaluronic acid using layer-layer self-assembly technology (Cui et al. 2010). The results showed that microcapsules can effectively kill bacteria, and the microcapsules will have a good application prospect in inhibiting drug release of bacterial infection. Chen et al. synthesized microcapsules embedded with doxorubicin hydrochloride (Chen et al. 2014). The microcapsules were composed of chitosan and heparin through layer by layer assembly, and the microcapsules could release doxorubicin hydrochloride under acidic conditions. However, none of these microcapsules is versatile.

This study aimed to develop the multifunctional fabric using green, facile and economical methods. Scheme 1 demonstrates the detail fabrication process of multifunctional fabric. Novel essence-loaded ethyecellulose/silica microcapsules were prepared by emulsion/solvent diffusion method, which were further recombined with waterborne resins to obtain multifunctional fabric coating. This coated fabric not only exhibits slow-release property of fragrance, but also has excellent superhydrophobicity and ultraviolet (UV) resistance. Due to the loaded essence and the modified silica of microcapsules, multifunctional fabric also demonstrated the durable superhydrophobicity and ultraviolet protection after being chemically or mechanically damaged. This fabric can be used in sports and fitness fabrics, safety protection fabrics and medical fabrics.

\section{Experimental}




\subsection{Materials}

3-isocyanatopropyltriethoxysilane (IPTS, 95\%), triethoxy-1H,1H,2H,2H-tridecafluoro-n-octylsilane (FAS, $>97 \%$ ), sodium dodecyl sulfate (SDS, $>98 \%$ ), acetic acid ( $\geq 99.7 \%$ ), ammonia solution $(25 \%)$ and ethyl acetate were obtained by Sinopharm Chemical Reagent Co., Ltd. (China). Tinuvin 1130 (T1130) was provided by BASF Chemical Co., Ltd. (Germany). Ethyecellulose (EC, $\geq 99 \%)$ was purchased from Zhengzhou Alfa Chemical Co., Ltd. (China). Dibutyltin dilaurate (DBTDL, $\geq 95 \%$ ) and tetraethoxysilane (TEOS, 98\%) were provided by Aladdin Chemical Reagent Co., Ltd. (China). Osmanthus flower fragrance $(\mathrm{OF}, \geq 99 \%)$ was offered by Henan Wanyong Biological Technology Co., Ltd. (China). Silicone waterproof emulsion (solid content: 15 wt\%) was provided by Jinan Xinglongda Chemical Co., Ltd. (China).

\subsection{Fabrication of Ethyecellulose/Silica Microcapsules (ESM)}

Appropriate T1130, DBTDL and IPTS were mixed with acetone in a $100 \mathrm{~mL}$ of round-bottom flask. The uniformly solution was magnetically stirred in the nitrogen atmosphere at $40^{\circ} \mathrm{C}$ for $4 \mathrm{~h}$ to obtain $\mathrm{T} 1130$ IPTS.

A solution of the EC and ethyl acetate was produced firstly. Then, $10 \mathrm{~g} \mathrm{EC}$ solution (solid content: $20 \%$ ), $1.5 \mathrm{OF}, 0.5 \mathrm{~g}$ FAS, $1 \mathrm{~g}$ TEOS and $0.5 \mathrm{~g}$ T1130-IPTS were mixed to form a uniform solution. The uniform solution was then added into $100 \mathrm{~g}$ saturated ethyl acetate aqueous solution with SDS to obtain the O/W emulsion under ultrasonic condition. Then, the obtained emulsion was poured into the $500 \mathrm{~g}$ deionized water under magnetically stirring for $12 \mathrm{~h}$ to obtain microcapsules solutions, and microcapsules were redistributed into water for further application. Ethyecellulose microcapsule without silica (EM) was fabricated as the above-mentioned process.

\subsection{Fabrication of multifunctional fabric coating}

Appropriate ESM emulsion (solid content: $20 \%$ ) was added into silicone waterproof emulsion to obtain a homogeneous coating under mechanically stirring $(500 \mathrm{rpm})$. Then, a piece of cotton was immersed into the coating for $5 \mathrm{~min}$, and then the coated cotton fabric was dried at $60^{\circ} \mathrm{C}$ for $15 \mathrm{~min}$. And other control fabric coatings were fabricated following the above-mentioned process.

\subsection{Characterization}

The optical microscope, the scanning electron microscope (SEM, QUANTA250, USA) and the transmission electron microscope (TEM, Tecnai G2F20, USA) were used to analyze the morphology of microcapsules was by The morphology of coatings was characterized the SEM (QUANTA250, USA). Elemental analysis and mapping were measured by energy dispersive spectroscope (EDS), which is attached to SEM. The FTIR of microcapsules was investigated by a FT-IR spectrometer (NICOLET, Thermo Fisher Scientific, China) with the wavenumber range from $500 \mathrm{~cm}^{-1}$ to $4000 \mathrm{~cm}^{-1}$. The thermal stability of microcapsules and control microcapsules were tested with thermo gravimetric analyzer (TGA,Q500,USA). Water contact angle (WCA) and sliding angle (SA) were characterized with an OCA15 contact angle analyzer (Data- 
physics, Germany). UV protection factor (UPF) value of the coating fabric and control fabric were tested by a UV protection tester (Darong,YGB 912E, China). UV classification was carried out according to AS/NZS 4399:1996. The washing stability of the coated fabric was tested on the basis of the standard washing process in AATCC 61-2003. The abrasion test of surfaces was performed by a homemade polish tester. The artificial ageing test of coatings was carried out by an accelerated aging tester (QUV/se, USA).

\section{Results And Discussion}

\subsection{Fabrication and characterization of ethyecellulose/silica composite microcapsules}

Multifunctional ethyecellulose/silica composite microcapsules were prepared by solvent extraction in one step. As shown in Fig. 1a and b, the optical micrograph shows that the prepared emulsion is spheroidal, and the obtained microcapsule emulsion has an obvious hollow structure. Further from TEM images (Fig. 1c and d), showed that the nuclei of microspheres were light and the shells were dark, which proved that the microspheres had a hollow structure. Further from SEM images of microcapsules, it can be found that the surface of microcapsule is rough with many small holes, and some silica particles are in the holes (Fig. 1e and 1f), which is mainly due to hydrolytic polycondensation of ethyl orthosilicate. Through EDS analysis, the surface of microcapsule contains C, O, Si and F chemical elements (Fig. 1g), illustrating that the shell of capsule is mainly composed of ethyecellulose and modified silica. In Fig. $1 \mathrm{~h}$, the absorption peaks at 2920 and $2850 \mathrm{~cm}^{-1}$ belong to the $-\mathrm{CH}_{3}$ and $-\mathrm{CH}_{2}$ - vibration, respectively, and a visible absorption peak at $3470 \mathrm{~cm}^{-1}$, which belongs to the stretching vibration of $-\mathrm{OH}$ of ethyecellulose and silica particles. Moreover, the typical absorption peak of FAS was located at $1199 \mathrm{~cm}^{-1}$, suggesting that FAS was successfully grafted onto the silica. In addition, there are visible absorption peaks at 1443 and $1373 \mathrm{~cm}^{-1}$ referred to $-\mathrm{C}-\mathrm{N}$, thereby illustrating the successful modification of T1130. Furthermore, the thermal stability of microcapsules was investigated by TGA. From Fig. $1 \mathrm{i}$ and $1 \mathrm{j}$, initial weight loss from $100^{\circ} \mathrm{C}-130^{\circ} \mathrm{C}$ is mainly due to the evaporation of water in the sample. The decomposition temperature of $\mathrm{OF}$ is between $130^{\circ} \mathrm{C}$ and $250^{\circ} \mathrm{C}$. However, a lot of weightlessness from $250^{\circ} \mathrm{C}$ to $370^{\circ} \mathrm{C}$ range is mainly caused by the degradation of the polymer ethyecellulose in microcapsule. The last weight loss may be owing to the degradation or polycondensation of silica nanoparticles. All these results also demonstrated that OF was successfully loaded into microcapsules.

\subsection{Fabrication and properties of multifunctional coating fabric}

When the mixture of hybrid microcapsules and waterborne polysiloxane resins was coated on the cotton fabric and cured to form the multifunctional fabric coating, the coated cotton fabric exhibits hierarchically rough surface compared with the smooth surface of pristine cotton or pure resins coated cotton, and the micro/nano-scale rough structures were mainly consisted of hybrid microcapsules, as shown in Fig. 2b-d. The water contact angle of coating increases with the increase of microcpasules 
content, When the microcapsule content is up to $45 \%$, the water contact angle of the coating reaches to $45 \mathrm{wt} \%$ (Fig. 2e). From Fig. 2f-2h, the surface of multifunctional coating was less tightly adhered to water droplets, and the water contact angle was $155^{\circ}$ and water droplets could easily roll from the inclined surface of the coated fabric $\left(S A=7.2^{\circ}\right)$. Furthermore, the surface of multifunctional coating could repel not only daily liquid droplets, such as coffee, soy sauce, juice and milk, but also red dye aqueous solution (Fig. 2i and 2j). Besides, the water flow could not wet the surface of multifunctional coating (Fig. 2k). All these results showed that this multifunctional fabric coating had the excellent superhydrophobicity. In addition, the coating always kept the superhydrophobicity, even though the surface of coated cotton was adhering to/pulling open (Fig. 2l), suggesting its high mechanical stability. This multifunctional coating fabric could repel water with $\mathrm{pH}$ value from 1 to 13 (Fig. $2 \mathrm{~m}$ ), indicating that it has the capacity for resistance of acid and alkali aqueous solution. Although this coating fabric was subjected to UV light irradiation, it could maintain the superhydrophobicity in contrast to the control coating without T1130 (Fig. 2n), which illustrates that this coated fabric exhibited good aging resistant ability. Most importantly, the fabric coating could sustain the superhydrophobicity after 6 times abrading/recovering cycles under heating to $80^{\circ} \mathrm{C}$ for $20 \mathrm{~min}$ (Fig. 20), suggesting that this fabric coating possessed the easily healing superhydrophobic ability.

\subsection{UV protection of multifunctional coating fabric}

In order to improve the UV protection of fabric, UV light absorbers T1130 were grafted onto the silica in the shell of microcapsules. When the UV absorbers were under sunlight, it absorbed the energy, and the chelate ring of UV absorbers was opened, so it can endow coating fabric with excellent UV protection, as shown in Fig. 3a. UPF values of cotton fabric based on the microcapsules were gradually increased along with the increase of T1130 content in the coating (Fig. 3b). The pristine cotton had low UPF values (10.05), suggesting that pristine cotton exhibited no UV protection ability. When the content of T1130 was up to $3 \mathrm{wt} \%$ in the fabric coatings, its UPF can reach to124.85, a data far beyond the efficient protection UPF rating (50+, Table 1). These results are corresponding to the UV transmittance values of the coated fabrics, which could come up to the minimum when the content of T1130 increased to $3 \%$ in the coating (Fig. 3c). As shown in Fig. 3d, the UPF value of the coating cotton still maintained the high UV protection even after 30 times dry friction. Moreover, since the coating cotton was washed for 10 times, the fabric could keep high UPF value (Fig. 3e), these results illustrate that the cotton coating based on microcapsules has the durable UV protection and could protect human body from UV light of sunlight for a long time. 
Table 1

UV protection factor of coated fabrics with different T1130

content.

\begin{tabular}{|lllll|}
\hline Samples & $\mathrm{T}_{(\mathrm{UV}-\mathrm{A})} / \%$ & $\mathrm{~T}_{(\mathrm{UV}-\mathrm{B})} / \%$ & $\mathrm{UPF}$ & Rating \\
\hline Control & 11.87 & 9.17 & 10.05 & $\mathrm{Bad}$ \\
\hline 0 wt\% T1130 & 10.87 & 7.17 & 11.91 & $\mathrm{Bad}$ \\
\hline 1 wt\% T1130 & 7.67 & 3.86 & 21.75 & $\mathrm{Bad}$ \\
\hline 2 wt\% T1130 & 3.84 & 1.1 & 71.87 & Good \\
\hline 3 wt\% T1130 & 3.31 & 0.61 & 124.85 & Excellent \\
\hline
\end{tabular}

\subsection{Fragrance releasing performance of coated cotton}

The releasing property and durability of microcapsules were further characterized. From Fig. 4a, the standard curve of OF is linearly fitted, and the linear regression equation is $C=0.056 \mathrm{~A}-0.005\left(R^{2}=0.9897\right)$, where $A$ and $C$ are the absorbance and concentration $(\mathrm{mg} / \mathrm{mL})$ of pure $O F$, respectively. This linear curve fits very well from the linear fitted equation. Compared with control microcapsules wihtout silica nanoparticles, hybrid microcapsules have the lower releasing rate of OF (19.4\%) even after 20 days (Fig. 4b), which is mainly due to the blocking of silica nanparticles to the pores of microcapsules. When these microcapsules were applied in the cotton, the treated cotton exhibited OF fragrance. However, the fragrance of fabric would gradually lose due to the volatilization of OF. The OF remnant rate of the cotton with OF is still high at the beginning, but after 15 days, the residue rate of OF dropped sharply to $5.2 \%$, indicating the OF-treated fabric is not long lasting. Compared with the OF-treated fabric, EM or ESM treated fabrics maintain a long-lasting fragrance. From Fig. 4c, the OF remnant rate of the cotton with ESM sustains $73.9 \%$ even after twenty days, and it is higher than the residual rate of EM-treated fabric (54.5\%), which is due to the shell of microcapsules slowed down the releasing of OF. Furthermore, the fragrance durability of fabric was evaluated by the washing test. After 4 washing cycles, there is only a $1.9 \%$ surplus of the cotton with OF. However, the OF residue rate of ESM-treated fabrics is higher, which remained above $26.4 \%$ even after 10 washing cycles (Fig. $4 \mathrm{~d}$ ). The results show that the ESM-treated fabric has good slow-release fragrance and washing resistance. In contrast, the residual amount OF in the fabric treated by microcapsule was higher, which remained above $30 \%$ OF after 10 times washing, because of the hydrogen bond interaction between microcapsules and cotton. It can be seen that the cotton fabric treated by microcapsules has the characteristics of slow-release and washable.

\section{Conclusion}

In conclusion, a multifunctional fabric coating based on OF-loaded ethyecellulose-silica composite microcapsules was successfully fabricated through a facile and green approach. The multifunctional ethyecellulose/silica microcapsules was firstly synthesised via emulsion-solvent diffusion method, in which OF was as the core of capsules and the FAS and UV absorber T1130 were grafted onto the silica in 
the shell of microcapsules. When these multifunctional microcapsules were dispersed into silicone resins to further form the fabric coating, the coated fabric displayed excellent superhydrophobicity (WCA: $155^{\circ}$; SA:7. $2^{\circ}$ ), slow-release property of fragrance, and UV protection (UPF:124.85). Most-importantly, coated fabric also possessed the durability for its multifunctions even after UV light irradiation, abrasion or washing. The multifunctional fabrics will further extend to clothing, home textiles, medical, military, cultural and sports, environmental protection, aerospace and other fabric field expansion, so it has become the top priority of fabric development.

\section{Declarations}

\section{Acknowledgements}

This work was financially supported by the Industry-University-Research Cooperation Projects (2021102614) and the School-Level Research Projects of Yancheng Institute of Technology (xjr2019030).

\section{Conflict of interest}

The authors declare that they have no conflict of interest.

\section{References}

Aracil MAB, Monllor P, Capablanca L, Gisbert J, Diaz P, Montava I (2015) A comparison between padding and bath exhaustion to apply microcapsules onto cotton. Cellulose 22:2117-2127.

Bilisik K, Demiryurek O (2012) Effects of sample dimensions on pull-out properties of woven fabric structures. Fibers Polym 13:1326-1334.

Bilisik K, Yildirim B (2014) Stick-slip properties of single and multiple yarn pull-out in dry and softening treated polyester satin woven fabrics in boundary region. Int J Cloth Sci Tech 26:67-95.

Cao FY, Yang B (2014) Supercooling suppression of microencapsulated phase change materials by optimizing shell composition and structure. Appl Energy 113:1512-1518.

Chen JY, Liang Y, Liu W, Huang J, Chen JH (2014) Fabrication of doxorubicin and heparin co-loaded microcapsules for synergistic cancer therapy. Int J Biol Macromol 69:554-560.

Cheng XQ, Wang ZX, Guo J, Ma J, Shao L (2018) Designing multifunctional coatings for cost-effectively sustainable water remediation. ACS Sustainable Chem Eng 6:1881-1890.

Chong YB, Sun D, Zhang X, Yue CY, Yang JL (2019) Robust multifunctional microcapsules with antibacterial and anticorrosion features. Chem Eng J 372:496-508.

Cui D, Szarpak A, Pignot-Paintrand I (2010) Contact-killing polyelectrolyte microcapsules based on chitosan derivatives. Adv Func Mater 20:3303-3312. 
Elesini US, Svarc J, Sumiga B, Urbas R (2017) Melamine formaldehyde microcapsules with fragrance core material: Preparation, properties, and end use. Text Res J 20:2435-2448.

Fei F, Wu YZ (2017) Photochromic properties of color-matching, double-shelled microcapsules covalently bonded onto cotton fabric and applications to outdoor clothing. J Appl Polym Sci 134;44698.

Han XT, Gong X (2021) In Situ, one-pot method to prepare robust superamphiphobic cotton fabrics for high buoyancy and good antifouling. ACS Appl Mater Inter 13:31298-31309.

Hong K, Park S (1999) Morphologies and release behavior of polyurea microcapsules from different polyisocyanates. J Mater Sci 34:3161-3164.

Hou JS, Yang YY, Yu DG et al (2021) Multifunctional fabrics finished using electrosprayed hybrid Janus particles containing nanocatalysts. Chem Eng J 411:128474.

Hu RM, Yang JL, Yang P et al (2020) Fabrication of ZnO@cotton fabric with anti-bacterial and radiation barrier properties using an economical and environmentally friendly method. Cellulose 27:2901-2911.

Hu Y, Yang Y, Ning Y, Wang CY, Tong Z (2013) Facile preparation of artemisia argyi oil-loaded antibacterial microcapsules by hydroxyapatite-stabilized Pickering emulsion templating. Colloids Surf $B$ 112:96-102.

lqbal K, Sun D (2014) Development of thermo-regulating polypropylene fibre containing microencapsulated phase change materials. Renew Energ 7l:473-479.

Jhatial AK, Khatri A, Ali S, Babar AA (2019) Sol-gel finishing of bamboo fabric with nanoparticles for water repellency, soil release and UV resistant characteristics. Cellulose 26:6365-6378.

Klemm D, Heublein B, Fink HP, Bohn A (2005) Cellulose: Fascinating biopolymer and sustainable raw material. Angew Chem Int Ed 44:3358-3393.

Lam PL, Lee KKH, Kok SHL et al (2012) Development of formaldehyde-free agar/gelatin microcapsules containing berberine $\mathrm{HCl}$ and gallic acid and their topical and oral applications. Soft Matter 8:5027-5037.

Liu HH, Wang Y, Li DM, Yan XF, Li RH (2019) Preparation and characterization of poly(melamineformaldehyde) microcapsules filled with propisochlor. J Macromol Sci A 56:676-685.

Li WH, Cai YR, Zhong QW, Yang Y, Kundu SC, Yao JM (2016) Silk sericin microcapsules with hydroxyapatite shells: protection and modification of organic microcapsules by biomimetic mineralization. J Mater Chem B 4:340-347.

Liu QZ, Yi C, Chen JH et al (2021) Flexible, breathable, and highly environmental-stable Ni/PPy/PET conductive fabrics for efficient electromagnetic interference shielding and wearable textile antennas. Compos B Eng 215:108725. 
Li QY, Zhang SY, Mahmood K et al (2021) Fabrication of multifunctional PET fabrics with flame retardant, antibacterial and superhydrophobic properties. Prog Org Coat 157:106296.

Lv LZ, Zhao WJ, Zhong XM, Fu, HQ (2020) Fabrication of magnetically inorganic/organic superhydrophobic fabrics and their applications. ACS Appl Mater Inter 12:45296-45305.

Medronho B, Lindman B (2015) Brief overview on cellulose dissolution/regeneration interactions and mechanisms. Adv Colloid Inter Sci 222:502-508.

Moreira ACG, Manrique YA, Martins IM et al (2020) Continuous production of melamine-formaldehyde microcapsules using a mesostructured reactor. Ind Eng Chem Res 59:18510-18519.

Pak AR, Park JH, Lee SG (2019) Blowing properties and functionality of thermoplastic polyester film using thermally expandable microcapsules. Polymers 11:1652.

Soares-Latour EM, Bernard J, Chambert S, Fleury E, Sintes-Zydowicz N (2013) Environmentally benign 100\% bio-based oligoamide microcapsules. Colloids Surf A Physicochem Eng Asp 524:193-203.

Song XX, Li HB, Tong WJ, Gao CY (2014) Fabrication of triple-labeled polyelectrolyte microcapsules for localized ratiometric pH sensing. J Colloid Interf Sci 416:252-257.

Son K, Yoo DI, Shin Y (2014) Fixation of vitamin E microcapsules on dyed cotton fabrics. Chem Eng J 239:284-289.

Sun G, Zhang Z (2001) Mechanical properties of melamine-formaldehyde microcapsules. J Microencapsul 18:593-602.

Taguchi Y, Yokoyama H, Kado H, Tanaka M (2007) Preparation of PCM microcapsules by using oil absorbable polymer particles. Colloids Surf A Physicochem Eng Asp 301:41-47.

Turan NY, Turker E, Insaatci O (2021) Microparticles loaded with propolis to make antibacterial cotton. Cellulose 28:4469-4483.

Wang HX, Niu HT, Zhou H, Wei X, Yang WD, Lin T (2019) Multifunctional directional water transport fabrics with moisture sensing capability, ACS Appl Mater Inter 11:22878-22884.

Wang SD, Wang K, Ma Q, Qu CX (2020) Fabrication of the multifunctional durable silk fabric with synthesized graphene oxide nanosheets. Mater Today Commun 23:100893.

Xie KL, Yu YH, Shi YQ (2009) Synthesis and characterization of cellulose/silica hybrid materials with chemical crosslinking. Carbohydr Polym 78:799-805.

Yang XF, Liu GQ, Peng L (2017) Highly efficient self-healable and dual responsive cellulose-based hydrogels for controlled release and 3D cell culture. Adv Funct Mater 27:1703174. 
Yuen CWM, Yip J, Liu LW et al (2012) Chitosan microcapsules loaded with either miconazole nitrate or clotrimazole, prepared via emulsion technique. Carbohyd Polym 89:795-801.

Zhao D, Jiao X, Zhang MM et al (2016) Preparation of high encapsulation efficiency fragrance microcapsules and their application in textiles. RSC Adv 6:80924-80933.

Zhao MX, Li MK,Wang L, Zhang X, Kong XF (2020) Preparation and characterization of Paraffin@CLPS/MS phase change microcapsules for thermal energy storage. ChemistrySelect 5:71907196.

Zhou XX, Song WF, Zhu GZ (2020) A facile approach for fabricating silica dioxide/reduced graphene oxide coated cotton fabrics with multifunctional properties. Cellulose 27: 2927-2938.

\section{Scheme}

Please see the Supplementary Files for the Scheme 1.

\section{Figures}



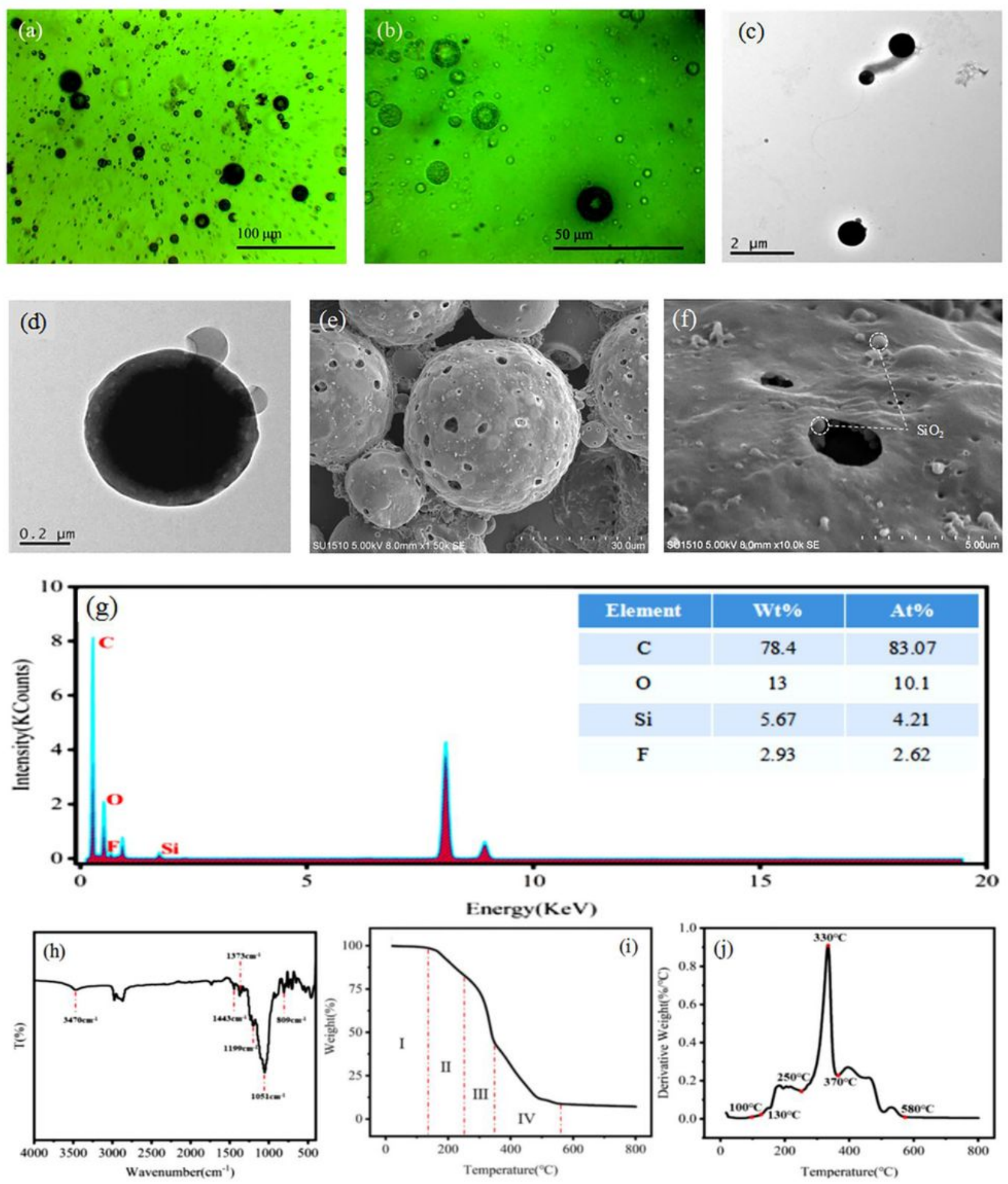

\section{Figure 1}

( $a$ and $b$ ) Optical images of the emulsion before and after ethyl acetate diffusion. (c) TEM image of ESM.

(d) The magnified TEM image of (c). (e) SEM image of ESM. (f) The magnified SEM image of (e). (g) EDS spectrum of ESM. (h) FT-IR spectra of ESM. ( $i$ and j) Thermogravimetric analysis (TGA and DTG) of ESM. 

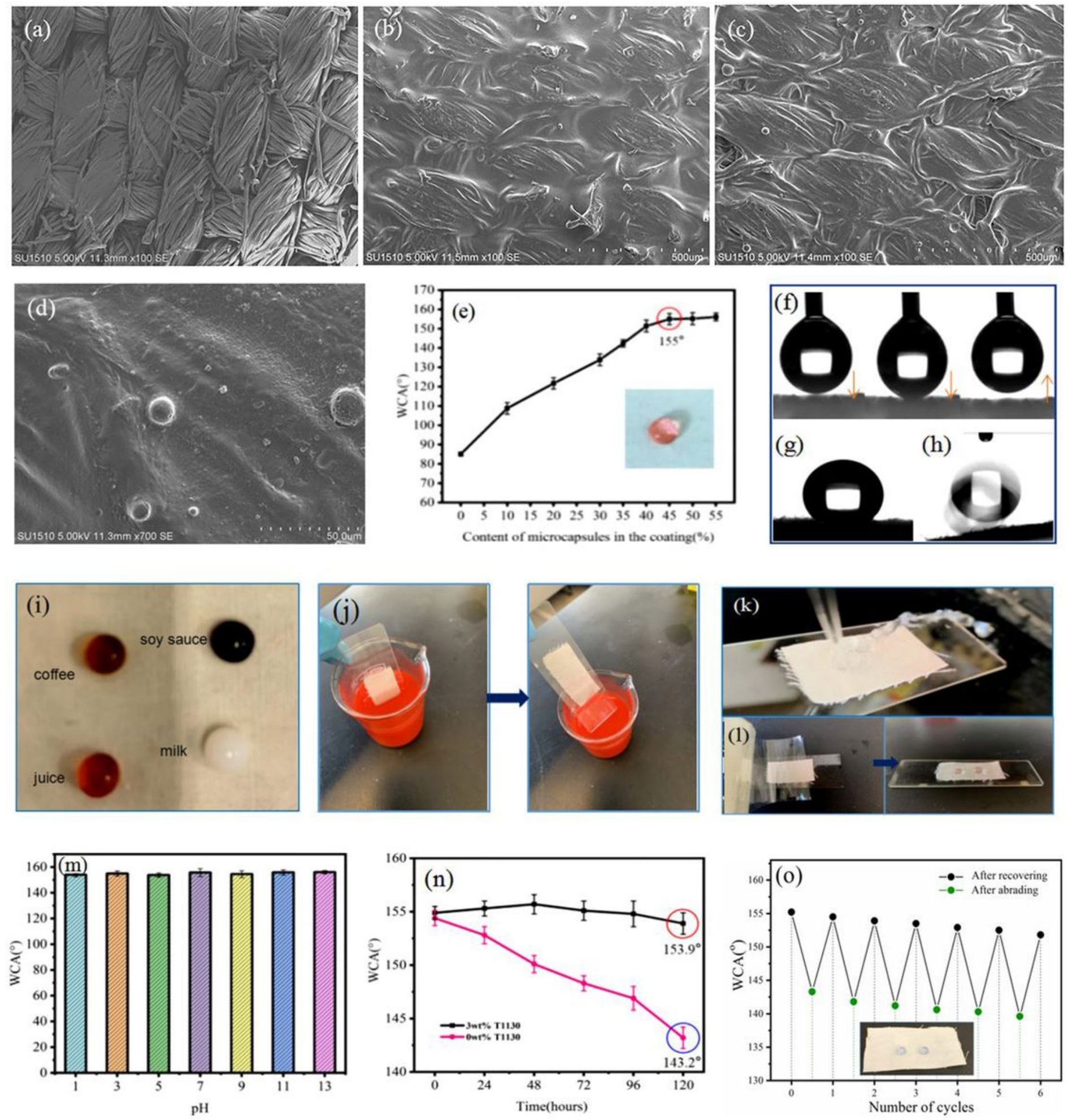

\section{Figure 2}

(a) SEM image of raw cotton fabric. (b) SEM image of the coated cotton fabric with pure resins. (c) The coated fabric with microcapsules/resins composite coating. (d) The magnified SEM image of (c). (e) Variations of WCA with different microcapsule content of coatings. (f) The superhydrophobic surface of coated fabric displayed the impermeability of water droplets. $(\mathrm{g})$ The photograph of water droplet on the superhydrophobic surface of coated fabric. (h) The photograph of water droplet rolling from the surface 
(SA=7.2). (i) The photograph of surface repelling daily liquid.droplets. (j) The photograph of surface repelling red dye aqueous solution. $(k)$ The photograph of surface non-wetting with water flow. (l) The photograph of water droplet on the taped surface of coated fabric. $(m)$ The superhydrophobic surface shows the good repellency for water droplets with different pH value from 1 to 13. (n) Variations of WCA with different contents of T1130 in the coatings under accelerated aging condition. (o) Variations of WCA several times abrading/recovering cycles.
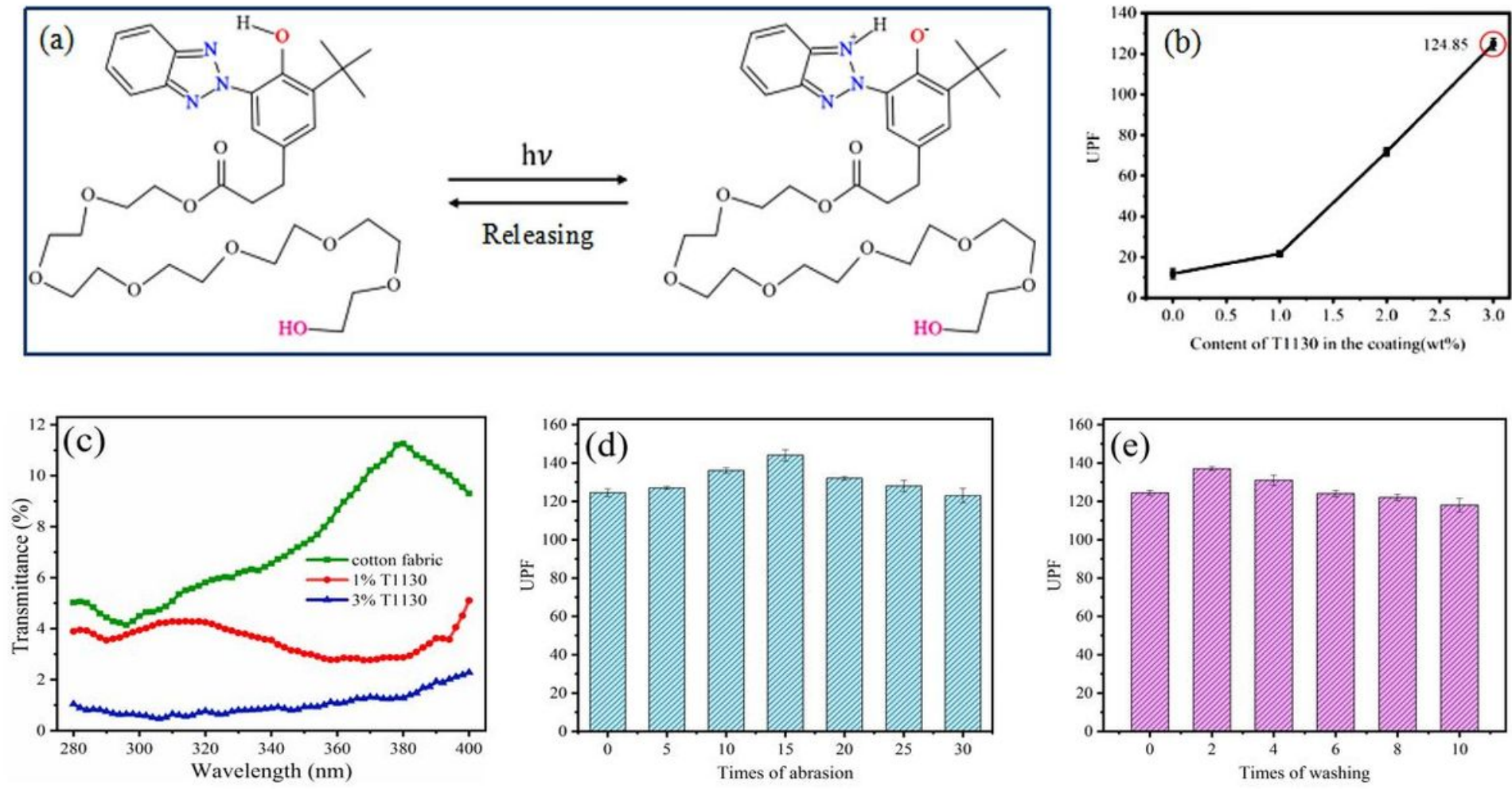

Figure 3

(a) Photostabilization mechanisms of T1130. (b) UPF changes of multifunctional coating fabric with different T1130 contents in the coating. (c) The transmittance spectra of textile coatings and cotton fabric. (d) UPF changes of multifunctional coating fabric along with different times of abrasion. (e) UPF changes of multifunctional coating fabric along with different times of washing. 

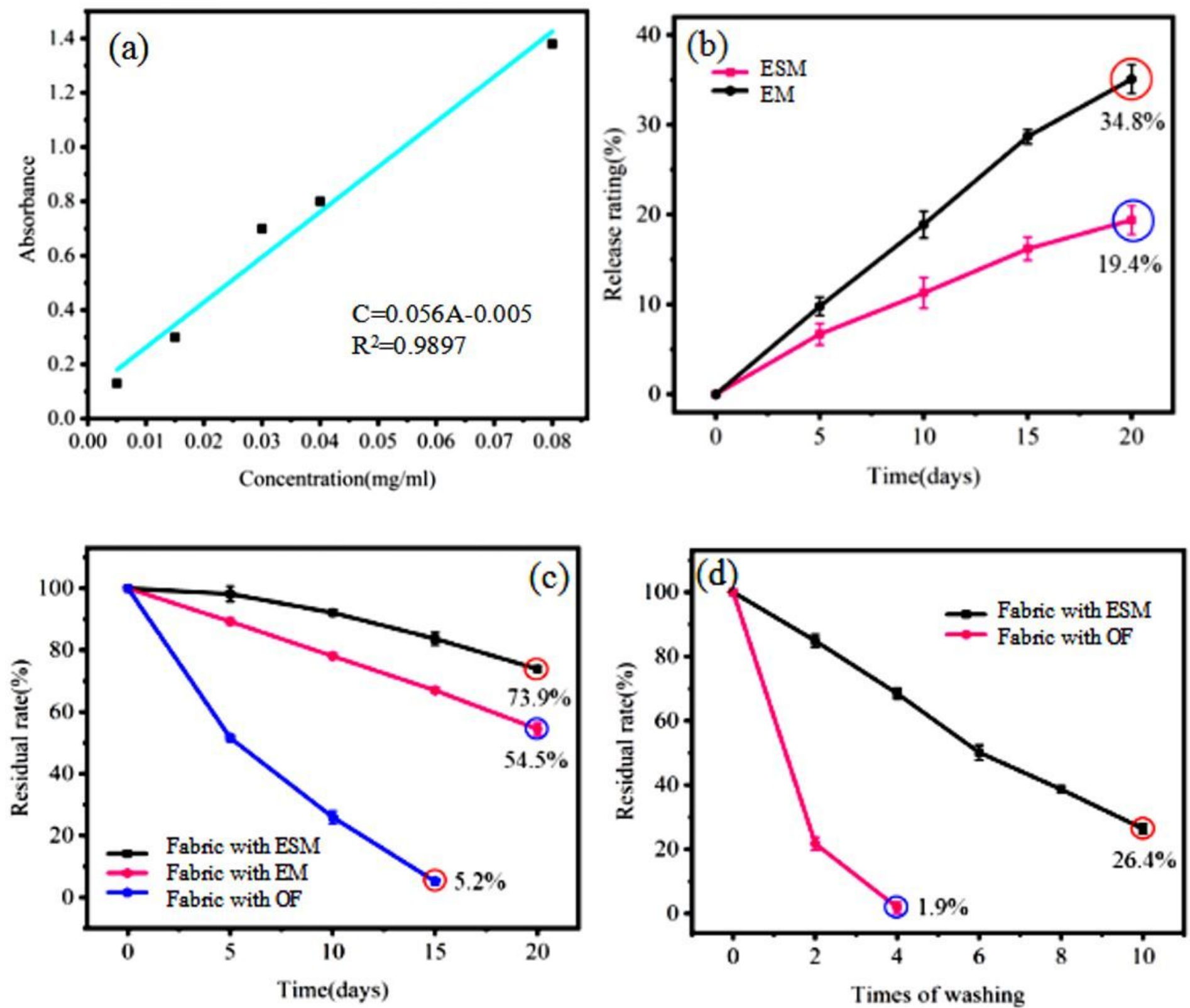

Figure 4

(a) The standard curve of OF. (b) The release properties of OF from EM-treated and ESM-treated cotton fabrics. (c) The OF releasing property of OF-treated, EM-treated and ESM-treated cotton fabrics under 25 oC condition. (b) The OF releasing property of OF-treated and ESM-treated cotton fabrics after repeated washing.

\section{Supplementary Files}

This is a list of supplementary files associated with this preprint. Click to download.

- scheme1.jpg

- graphicsabstract.jpg 